\title{
Do people change their diet after colorectal adenoma diagnosis?
}

\author{
$\underline{\text { Abeir El MOgassabi }}{ }^{1}$, Elizabeth Williams ${ }^{1}$, Bernard Corfe $^{1}$ and Mark Hull ${ }^{2}$ \\ ${ }^{1}$ The University of Sheffield, Sheffield, United Kingdom and \\ ${ }^{2}$ University of Leeds, Leeds, United Kingdom
}

\section{Abstract}

Colorectal cancer (CRC) is the fourth most common cancer in the UK; 95\% of CRCs develop from colorectal adenomas. Adults in England aged 60-74 years are screened for colorectal cancer through the national Bowel Cancer Screening Programme (BCSP) which aims to detect colorectal cancer early, and also remove colorectal adenomas. However, adenoma recurrence is common. The World Cancer Research Fund (WCRF) estimates that around $45 \%$ of CRC cases could be prevented by a healthy lifestyle. There are no specific dietary guidelines to protect against adenoma recurrence and patients do not receive dietary advice in the care pathway. Nonetheless understanding the dietary intake of people with adenoma who are at high-risk of developing CRC and their subsequent dietary behaviour post-diagnosis may design future intervention strategies.

This study aims to describe the diet of colorectal adenoma patients and evaluate whether there were elective changes in diet following diagnosis.

The data used in this study were obtained from colorectal adenoma patients at high risk of recurrence, recruited to the seAFOod polyp prevention trial through the English BCSP. Dietary intake was assessed using the EPIC Food Frequency Questionnaire at two time points: during the 12 months before and during the 12 months after adenoma diagnosis. FETA dietary analysis software was used to extract data. Paired Sample-T-Tests and Wilcoxon Signed-Rank Test were used to assess change in intake using SPSS version 25.

Of the 709 patients recruited to the main study, 526 completed the FFQ on both occasions, of whom $81.7 \%$ were males. The mean age was 65 years (SD 4.8), 83.4\% were overweight or obese with mean BMI $=29.5 \mathrm{~kg} / \mathrm{m}^{2}$ (SD 5.7). The majority of nutrient intakes met UK DRVs, with the exception of low intake of fibre, vitamin D and selenium. Following diagnosis, a significant reduction was detected in daily intake of energy (from 7.7 to $7.5 \mathrm{MJ}, p<0.05$ ) meat and meat products (from 124 to $111 \mathrm{~g}, p<0.005$ ), non-alcoholic beverages (from 939 to $898 \mathrm{~g}, p<0.05$ ) and nuts (from 5.5 to $4.3 \mathrm{~g}, p<0.05$ ). Subgroup analysis revealed that dietary changes were confined to men where the reduction in energy was by $293 \mathrm{kj} / \mathrm{day}$ and in meat by 1.5 portions per week.

Patients do modify diet following a diagnosis of adenoma and changes correspond to WCRF healthy diet guidelines. Whether detected difference between males and females was due to the sample size or due to actual difference in behavior is not clear.

\section{Conflict of Interest}

There is no conflict of interest 